\title{
Positivity in coefficient-free rank two cluster algebras
}

\author{
G. Dupont \\ Université de Lyon \\ Université Lyon 1 \\ Institut Camille Jordan \\ 43, Bd du 11 novembre 1918 \\ F-69622 Villeurbanne cedex \\ dupont@math . univ-lyon1.fr
}

Submitted: Jan 30, 2009; Accepted: Jul 30, 2009; Published: Aug 7, 2009

Mathematics Subject Classification: 13F60, 16G20, 05E10, 05E40

\begin{abstract}
Let $b, c$ be positive integers, $x_{1}, x_{2}$ be indeterminates over $\mathbb{Z}$ and $x_{m}, m \in \mathbb{Z}$ be rational functions defined by $x_{m-1} x_{m+1}=x_{m}^{b}+1$ if $m$ is odd and $x_{m-1} x_{m+1}=x_{m}^{c}+1$ if $m$ is even. In this short note, we prove that for any $m, k \in \mathbb{Z}, x_{k}$ can be expressed as a substraction-free Laurent polynomial in $\mathbb{Z}\left[x_{m}^{ \pm 1}, x_{m+1}^{ \pm 1}\right]$. This proves FominZelevinsky's positivity conjecture for coefficient-free rank two cluster algebras.
\end{abstract}

\section{Introduction}

\section{A combinatorial result}

Let $b, c$ be positive integers and $x_{1}, x_{2}$ be indeterminates over $\mathbb{Z}$. The (coefficient-free) cluster algebra $\mathcal{A}(b, c)$ is the subring of the field $\mathbb{Q}\left(x_{1}, x_{2}\right)$ generated by the elements $x_{m}, m \in \mathbb{Z}$ satisfying the recurrence relations:

$$
x_{m+1}= \begin{cases}\frac{x_{m}^{b}+1}{x_{m-1}} & \text { if } m \in 2 \mathbb{Z}+1 \\ \frac{x_{m}^{c}+1}{x_{m-1}} & \text { if } m \in 2 \mathbb{Z}\end{cases}
$$

The elements $x_{m}, m \in \mathbb{Z}$ are called the cluster variables of $\mathcal{A}(b, c)$ and the pairs $\left(x_{m}, x_{m+1}\right), m \in \mathbb{Z}$ are called the clusters of $\mathcal{A}(b, c)$.

The Laurent phenomenon [FZ02] implies that for any $m \in \mathbb{Z}$ and any $k \in \mathbb{Z}$ the cluster variable $x_{k}$ belongs to the ring of Laurent polynomials $\mathbb{Z}\left[x_{m}^{ \pm 1}, x_{m+1}^{ \pm 1}\right]$.

When $b c \leqslant 4$, it was proved by Sherman-Zelevinsky [SZ04] and independently by Musiker-Propp [MP06] that for any $m \in \mathbb{Z}$ and any $k \in \mathbb{Z}$ the cluster variable $x_{k}$ belongs 
to $\mathbb{N}\left[x_{m}^{ \pm 1}, x_{m+1}^{ \pm 1}\right]$. This was later proved by Caldero-Reineke for any $b=c$ [CR08]. In this paper, we prove this for arbitrary positive integers $b, c$. More precisely, the main result of the paper is :

Theorem 8. Let b, c be positive integers. With the above notations, we have

$$
\left\{x_{k}: k \in \mathbb{Z}\right\} \subset \mathbb{N}\left[x_{m}^{ \pm 1}, x_{m+1}^{ \pm 1}\right]
$$

for any $m \in \mathbb{Z}$.

\section{The positivity conjecture for cluster algebras}

In particular, this result is a particular case of a general conjecture formulated by Fomin and Zelevinsky for arbitrary cluster algebras. We recall that cluster algebras were introduced by Fomin and Zelevinsky in a series of papers [FZ02, FZ03, BFZ05, FZ07] in order to design a general framework for understanding total positivity in algebraic groups and canonical bases in quantum groups. They turned out to be related to various subjects in mathematics like combinatorics, Lie theory, representation theory, Teichmüller theory and many other topics.

In full generality, a (coefficient-free) cluster algebra is a commutative algebra generated by indeterminates over $\mathbb{Z}$ called cluster variables. They are gathered into sets of fixed cardinality called clusters. The initial data for constructing a (coefficient-free) cluster algebra is a seed, that is, a pair $(B, \mathbf{u})$ where $B \in M_{q}(\mathbb{Z})$ is a skew-symmetrizable matrix and $\mathbf{u}=\left(u_{1}, \ldots, u_{q}\right)$ is a $q$-tuple of indeterminates over $\mathbb{Z}$. The cluster variables are defined inductively by a process called mutation. The cluster algebra associated to a seed $(B, \mathbf{u})$ is denoted by $\mathcal{A}(B)$. For every cluster $\mathbf{c}=\left\{c_{1}, \ldots, c_{q}\right\}$ in $\mathcal{A}(B)$, the Laurent phenomenon ensures that the cluster algebra $\mathcal{A}(B)$ is a $\mathbb{Z}$-subalgebra of the ring $\mathbb{Z}\left[c_{1}^{ \pm 1}, \ldots, c_{q}^{ \pm 1}\right]$ of Laurent polynomials in c [FZ02].

A Laurent polynomial is called substraction-free if it can be written as a $\mathbb{N}$-linear combination of Laurent monomials. In [FZ02], Fomin and Zelevinsky gave the so-called positivity conjecture for arbitrary cluster algebras. In the coefficient-free case, this conjecture can be stated as follows:

Conjecture 1 ([FZ02]). Let $\mathcal{A}$ be a cluster algebra. Then any cluster variable $x$ in $\mathcal{A}$ can be written as a substraction-free Laurent polynomial in any cluster of $\mathcal{A}$.

A simple but non-trivial class of cluster algebras is constituted by the so-called rank two cluster algebras, that is, the cluster algebras of the form $\mathcal{A}\left(B_{b, c}\right)$ with

$$
B_{b, c}=\left[\begin{array}{rr}
0 & b \\
-c & 0
\end{array}\right] \in M_{2}(\mathbb{Z}) .
$$

Note that $\mathcal{A}\left(B_{b, c}\right)$ is the cluster algebra $\mathcal{A}(b, c)$ introduced at the beginning.

Thus, Theorem 8 is equivalent to the following statement:

Theorem. Let $b, c$ be positive integers. Then Conjecture 1 holds for the cluster algebra $\mathcal{A}(b, c)$. 


\section{Organization of the paper}

Despite the fact the main theorem can be expressed in purely combinatorial terms, the methods we use in this paper are based on representation theory and more precisely on categorifications of cluster algebras using cluster categories and cluster characters developed in $\left[\mathrm{BMR}^{+}\right.$06, CC06, CK08, CK06]. Positivity will follow from results of Caldero-Reineke [CR08] and folding processes inspired by methods in [Dup08] (see also [Dem08b, Dem08a]). In section 1, we recall the necessary background on cluster categories and cluster characters. In section 2 , we investigate briefly cluster categories and cluster algebras associated to quivers with automorphisms. In section 3, we define a folding process in order to realize cluster variables in rank two cluster algebras and we prove the main result.

\section{Cluster categories and cluster characters}

In this section, we recall necessary background on cluster categories and cluster characters. In the whole paper, $k$ will denote the field $\mathbb{C}$ of complex numbers. Let $Q=\left(Q_{0}, Q_{1}\right)$ be a finite acyclic quiver where $Q_{0}$ is the set of vertices and $Q_{1}$ the set of arrows. For an arrow $\alpha: i \longrightarrow j \in Q_{1}$, we denote by $s(\alpha)=i$ its source and $t(\alpha)=j$ its target. The fact that $Q$ is acyclic means that $Q$ contains no oriented cycles.

To the quiver $Q$, we can associate a skew-symmetric matrix $B_{Q}=\left(b_{i j}\right)_{i, j \in Q_{0}}$ as follows:

$$
b_{i j}=\mid\left\{\alpha \in Q_{1}: s(\alpha)=i \text { and } t(\alpha)=j\right\}|-|\left\{\alpha \in Q_{1}: t(\alpha)=i \text { and } s(\alpha)=j\right\} \mid .
$$

This induces a 1-1 correspondence from the set of quivers without loops and 2-cycles to the set of skew-symmetric matrices.

\subsection{Cluster categories}

A representation $V$ of $Q$ is a pair

$$
V=\left((V(i))_{i \in Q_{0}},(V(\alpha))_{\alpha \in Q_{1}}\right)
$$

where $(V(i))_{i \in Q_{0}}$ is a family of finite dimensional $k$-vector spaces and $(V(\alpha))_{\alpha \in Q_{1}}$ is a family of $k$-linear maps $V(\alpha): V(s(\alpha)) \longrightarrow V(t(\alpha))$. A morphism of representations $f$ : $V \longrightarrow W$ is a family $\left(f_{i}\right)_{i \in Q_{0}}$ of $k$-linear maps such that the following diagram commutes

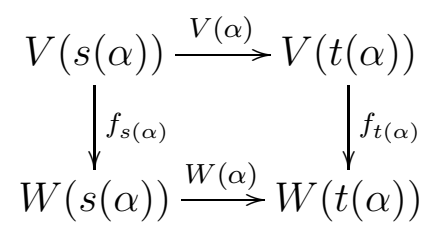

for any arrow $\alpha \in Q_{1}$. This defines a category $\operatorname{rep}(Q)$ which is equivalent to the category $k Q$-mod of finitely generated left-modules over the path algebra $k Q$ of $Q$. 
For any vertex $i \in Q_{0}$, we denote by $P_{i}$ (resp. $I_{i}, S_{i}$ ) the indecomposable projective (resp. injective, simple) $k Q$ module associated to the vertex $i$. For any representation $M$ of $Q$, the dimension vector of $M$ is the vector $\operatorname{dim} M=(\operatorname{dim} M(i))_{i \in Q_{0}} \in \mathbb{N}^{Q_{0}}$.

We denote by $D^{b}(k Q)$ the bounded derived category of $Q$. This is a triangulated category with shift functor [1] and Auslander-Reiten translation $\tau$. The cluster category of $Q$, introduced in $\left[\mathrm{BMR}^{+} 06\right]$, is the orbit category $\mathcal{C}_{Q}=D^{b}(k Q) / \tau^{-1}[1]$ of the autofunctor $\tau^{-1}[1]$ in $D^{b}(k Q)$. This is a triangulated category [Kel05]. Moreover, it is proved in $\left[\mathrm{BMR}^{+} 06\right]$ that indecomposable objects are given by

$$
\operatorname{ind}\left(\mathcal{C}_{Q}\right)=\operatorname{ind}(k Q-\bmod ) \sqcup\left\{P_{i}[1]: i \in Q_{0}\right\}
$$

and that $\mathcal{C}_{Q}$ is a 2-Calabi-Yau category, that is, that there is a bifunctorial duality

$$
\operatorname{Ext}_{\mathcal{C}_{Q}}^{1}(M, N) \simeq D \operatorname{Ext}_{\mathcal{C}_{Q}}^{1}(N, M)
$$

for any two objects $M, N$ in the cluster category where $D=\operatorname{Hom}_{k}(-, k)$.

\subsection{The Caldero-Chapoton map}

We denote by $\langle-,-\rangle$ the homological Euler form on $k Q$-mod. For any representation $M \in \operatorname{rep}(Q)$ and any $\mathbf{e} \in \mathbb{N}^{Q_{0}}$, the grassmannian of submodules of $M$ is the projective variety

$$
\operatorname{Gr}_{\mathbf{e}}(M)=\{N \text { subrepresentation of M s.t. } \operatorname{dim} N=\mathbf{e}\} .
$$

We can thus consider the Euler-Poincaré characteristic $\chi\left(\operatorname{Gr}_{\mathbf{e}}(M)\right)$ of this variety.

We denote by $\mathcal{A}(Q)$ the coefficient-free cluster algebra with initial seed $\left(B_{Q}, \mathbf{u}\right)$ where $\mathbf{u}=\left\{u_{i}: i \in Q_{0}\right\}$ is a set of indeterminates over $\mathbb{Q}$. In [CC06], the authors considered a map $X_{\text {? }}: \operatorname{Ob}\left(\mathcal{C}_{Q}\right) \longrightarrow \mathbb{Z}\left[\mathbf{u}^{ \pm 1}\right]$ which is now referred to as the Caldero-Chapoton map (also called cluster character in the literature).

Definition 2. The Caldero-Chapoton map is the map $X_{\text {? }}$ defined from the set of objects in $\mathcal{C}_{Q}$ to the ring of Laurent polynomials in the indeterminates $\left\{u_{i}, i \in Q_{0}\right\}$ by:

a. If $M$ is an indecomposable $k Q$-module, then

$$
X_{M}=\sum_{\mathbf{e} \in \mathbb{N}^{Q_{0}}} \chi\left(\operatorname{Gr}_{\mathbf{e}}(M)\right) \prod_{i \in Q_{0}} u_{i}^{-\left\langle\mathbf{e}, \operatorname{dim} S_{i}\right\rangle-\left\langle\operatorname{dim} S_{i}, \operatorname{dim} M-\mathbf{e}\right\rangle} ;
$$

b. If $M=P_{i}[1]$ is the shift of the projective module associated to $i \in Q_{0}$, then

$$
X_{M}=u_{i}
$$

c. For any two objects $M, N$ in $\mathcal{C}_{Q}$,

$$
X_{M \oplus N}=X_{M} X_{N}
$$


Note that equality (1) also holds for decomposable modules.

One of the main motivations for introducing the Caldero-Chapoton map was:

Theorem 3 ([CK06]). Let $Q$ be an acyclic quiver. Then $X_{\text {? }}$ induces a 1-1 correspondence from the set of indecomposable objects without self-extensions in $\mathcal{C}_{Q}$ and cluster variables in $\mathcal{A}(Q)$.

Moreover, it gives the cluster algebra $\mathcal{A}(Q)$ a structure of Hall algebra of the cluster category $\mathcal{C}_{Q}$. More precisely, Caldero and Keller proved the following:

Theorem 4 ([CK06]). Let $Q$ be an acyclic quiver, $M, N$ be two objects in $\mathcal{C}_{Q}$ such that $\operatorname{Ext}_{\mathcal{C}_{Q}}^{1}(M, N) \simeq k$, then

$$
X_{M} X_{N}=X_{B}+X_{B^{\prime}}
$$

where $B$ and $B^{\prime}$ are the unique objects such that there exists non-split triangles

$$
M \longrightarrow B \longrightarrow N \longrightarrow M[1] \text { and } N \longrightarrow B^{\prime} \longrightarrow M \longrightarrow N[1]
$$

Caldero and Reineke later proved an important result towards positivity:

Theorem 5 ([CR08]). Let $Q$ be an acyclic quiver, $M$ be an indecomposable module, then $\chi\left(\operatorname{Gr}_{e}(M)\right) \geqslant 0$.

In particular, this result combined with Theorem 3 proves that if $Q$ is an acyclic quiver, cluster variables in $\mathcal{A}(Q)$ can be written as substraction-free Laurent polynomials in the initial cluster.

\section{Automorphisms of quivers}

Let $Q=\left(Q_{0}, Q_{1}\right)$ be an acyclic quiver and $B_{Q}=\left(b_{i j}\right) \in M_{Q_{0}}(\mathbb{Z})$ be the associated matrix.

A subgroup $G$ of the symmetric group $\mathfrak{S}_{Q_{0}}$ is called a group of automorphisms of $Q$ if $b_{g i, g j}=b_{i, j}$ for any $i, j \in Q_{0}$ and any $g \in G$.

\section{$2.1 \quad G$-action on the cluster category}

Fix an acyclic quiver $Q$ equipped with a group of automorphisms $G$. We define a group action of $G$ on $\operatorname{rep}(Q)$ as follows. For any $g \in G$ and any representation $V$, set $g V$ to be the representation $\left(\left(V\left(g^{-1} i\right)\right)_{i \in Q_{0}},\left(V\left(g^{-1} \alpha\right)\right)_{\alpha \in Q_{1}}\right)$. For any morphism of representation $f: V \longrightarrow W, g f$ is the morphism $g V \longrightarrow g W$ given by $g f=\left(f_{g^{-1}}\right)_{i \in Q_{0}}$. Each $g$ defines a $k$-linear auto-equivalence of $\operatorname{rep}(Q)$ with quasi-inverse $g^{-1}$. The group $G$ also acts on $\mathbb{N}^{Q_{0}}$ by $g . \mathbf{e}=\left(e_{g^{-1} i}\right)_{i \in Q_{0}}$ for any $\mathbf{e}=\left(e_{i}\right)_{i \in Q_{0}} \in \mathbb{N}^{Q_{0}}$ and any $g \in G$.

Each $g$ induces an action by auto-equivalence on the bounded derived category $D^{b}(k Q)$ commuting with the shift functor [1] and the Auslander-Reiten translation $\tau$. Thus, each $g \in G$ induces an action by auto-equivalence on the cluster category $\mathcal{C}_{Q}=D^{b}(k Q) / \tau^{-1}[1]$. This action is additive and given on shift of projective objects by $g P_{i}[1] \simeq P_{g i}[1]$ for any $g \in G$ and $i \in Q_{0}$. 


\section{$2.2 \quad G$-action and variables}

We still consider an acyclic quiver $Q$ endowed with a group of automorphisms $G$. Let $\mathcal{A}(Q)$ be the coefficient-free cluster algebra with initial seed $\left(B_{Q}, \mathbf{u}\right)$ with $\mathbf{u}=\left\{u_{i}: i \in Q_{0}\right\}$. We define an action of $G$ by $\mathbb{Z}$-algebra homomorphisms on the ring of Laurent polynomials $\mathbb{Z}\left[\mathbf{u}^{ \pm 1}\right]$ by setting $g u_{i}=u_{g i}$.

The following lemma was already proved in [Dup08], we give the proof for completeness.

Lemma 6. Let $Q$ be an acyclic quiver equipped with a group $G$ of automorphisms. Then for any object $M$ in $\mathcal{C}_{Q}$, we have

$$
g X_{M}=X_{g M}
$$

Proof. Let $M$ be an object in $\mathcal{C}_{Q}$. If $M$ is not indecomposable, we write $M=\bigoplus_{i} M_{i}$ with each $M_{i}$ being indecomposable. For any $g \in G$, we have

$$
X_{g M}=X_{g\left(\oplus_{i} M_{i}\right)}=X_{\bigoplus_{i} g M_{i}}=\prod_{i} X_{g M_{i}} .
$$

Since the $G$ acts on $\mathbb{Z}\left[\mathbf{u}^{ \pm 1}\right]$ by morphisms of $\mathbb{Z}$-algebras, we can thus assume that $M$ is indecomposable.

For any $i \in Q_{0}$ and any $g \in G$, we have $X_{g P_{i}[1]}=g u_{i}=u_{g i}=X_{P_{g i}[1]}$, thus, it is enough to prove the lemma for indecomposable $k Q$-modules. Since $G$ acts by auto-equivalences on $k Q$-mod, the homological Euler form is $G$-invariant, that is, $\langle g \mathbf{e}, g \mathbf{f}\rangle=\langle\mathbf{e}, \mathbf{f}\rangle$ for any $\mathbf{e}, \mathbf{f} \in \mathbb{N}^{Q_{0}}$ and any $g \in G$. Moreover, the action of $g$ induces an isomorphism of varieties $\operatorname{Gr}_{\mathbf{e}}(M) \simeq \operatorname{Gr}_{g \mathbf{e}}(g M)$ for any $\mathbf{e} \in \mathbb{N}^{Q_{0}}$ and any $g \in G$. We thus have

$$
\begin{aligned}
g X_{M} & =\sum_{\mathbf{e} \in \mathbb{N}^{Q_{0}}} \chi\left(\operatorname{Gr}_{e}(M)\right) \prod_{i \in Q_{0}} u_{g i}^{-\left\langle\operatorname{dim} M, \operatorname{dim} S_{i}\right\rangle-\left\langle\operatorname{dim} S_{i}, \operatorname{dim} M-\mathbf{e}\right\rangle} \\
& =\sum_{\mathbf{e} \in \mathbb{N}^{Q_{0}}} \chi\left(\operatorname{Gr}_{e}(M)\right) \prod_{i \in Q_{0}} u_{i}^{-\left\langle\operatorname{dim} M, \operatorname{dim} S_{g^{-1}}\right\rangle-\left\langle\operatorname{dim} S_{g^{-1}}, \operatorname{dim} M-\mathbf{e}\right\rangle}
\end{aligned}
$$

and

$$
\begin{aligned}
X_{g M} & =\sum_{\mathbf{e} \in \mathbb{N}^{Q_{0}}} \chi\left(\operatorname{Gr}_{\mathbf{e}}(g M)\right) \prod_{i \in Q_{0}} u_{i}^{-\left\langle g \operatorname{dim} M, \operatorname{dim} S_{i}\right\rangle-\left\langle\operatorname{dim} S_{i}, g \operatorname{dim} M-\mathbf{e}\right\rangle} \\
& =\sum_{\mathbf{e} \in \mathbb{N}^{Q_{0}}} \chi\left(\operatorname{Gr}_{g^{-1} \mathbf{e}}(M)\right) \prod_{i \in Q_{0}} u_{i}^{-\left\langle\operatorname{dim} M, \operatorname{dim} S_{g^{-1}}\right\rangle-\left\langle\operatorname{dim} S_{g^{-1}}, \operatorname{dim} M-g^{-1} \mathbf{e}\right\rangle} \\
& =\sum_{\mathbf{e} \in \mathbb{N}^{Q_{0}}} \chi\left(\operatorname{Gr}_{\mathbf{e}}(M)\right) \prod_{i \in Q_{0}} u_{i}^{-\left\langle\operatorname{dim} M, \operatorname{dim} S_{g^{-1}}\right\rangle-\left\langle\operatorname{dim} S_{g^{-1}}, \operatorname{dim} M-\mathbf{e}\right\rangle} \\
& =g X_{M} .
\end{aligned}
$$




\section{Unfolding rank two cluster algebras}

The main idea of this paper comes from folding processes first developed by the author in [Dup08]. Fix $b, c$ two positive integers and $\mathbf{v}=\left\{v_{1}, \ldots, v_{b}\right\}, \mathbf{w}=\left\{w_{1}, \ldots, w_{c}\right\}$ two finite sets. Let $K_{b, c}$ be the quiver given by

$$
\left(K_{b, c}\right)_{0}=\mathbf{v} \sqcup \mathbf{w}
$$

and for every $i \in\{1, \ldots, b\}, j \in\{1, \ldots, c\}$, there is exactly one arrow $v_{i} \longrightarrow w_{j}$. The quiver $K_{b, c}$ can be represented as follows:

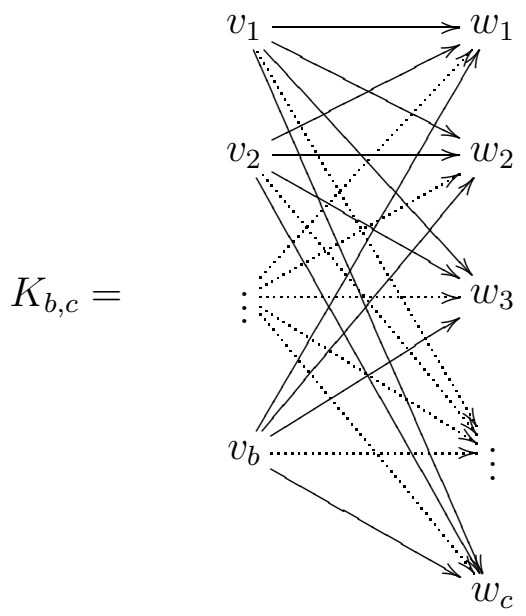

Note that $K_{b, c}$ is acyclic and that the group

$$
G=\mathfrak{S}_{\mathbf{v}} \times \mathfrak{S}_{\mathbf{w}}
$$

is a group of automorphisms for $K_{b, c}$.

In order to simplify notations, we write $Q=K_{b, c}$. We denote by $\mathcal{A}(Q)$ the coefficientfree cluster algebra with initial seed $\left(\mathbf{u}, B_{Q}\right)$ where

$$
\mathbf{u}=\left\{u_{v_{1}}, \ldots, u_{v_{b}}, u_{w_{1}}, \ldots, u_{w_{c}}\right\} .
$$

We define the following $\mathbb{Z}$-algebra homomorphism $\pi$ called folding:

$$
\pi:\left\{\begin{array}{rrrrr}
\mathbb{Z}\left[u_{i}^{ \pm 1}: i \in Q_{0}\right] & \longrightarrow \mathbb{Z}\left[x_{1}^{ \pm 1}, x_{2}^{ \pm 1}\right] & \\
u_{v_{i}} & \mapsto x_{1} & \text { for all } i=1, \ldots, b ; \\
u_{w_{i}} & \mapsto x_{2} & & \text { for all } i=1, \ldots, c .
\end{array}\right.
$$

We will denote by $P_{\mathbf{v}}$ (resp. $\left.P_{\mathbf{w}}\right)$ a representative of the set $\left\{P_{v_{1}}, \ldots, P_{v_{b}}\right\}$ (resp. $\left.\left\{P_{w_{1}}, \ldots, P_{w_{c}}\right\}\right)$.

We denote by $X_{\text {? }}$ the Caldero-Chapoton map on $\mathcal{C}_{Q}$. For every $g \in G$ and $i \in \mathbb{Z}$, we have $g P_{\mathbf{v}}[i]=g P_{v^{\prime}}[i] \in\left\{P_{v_{1}}[i], \ldots, P_{v_{b}}[i]\right\}$ for some $v^{\prime} \in \mathbf{v}$ and $g P_{\mathbf{w}}[i]=g P_{w^{\prime}}[i] \in$ $\left\{P_{w_{1}}[i], \ldots, P_{w_{c}}[i]\right\}$ for some $w^{\prime} \in \mathbf{w}$. It follows from Lemma 6 that $g X_{P_{\mathbf{v}}[i]}=X_{g P_{\mathbf{v}}[i]}=$ $X_{P_{v^{\prime}}[i]}$ (resp. $\left.g X_{P_{\mathbf{w}}[i]}=X_{g P_{\mathbf{w}}[i]}=X_{P_{w^{\prime}}[i]}\right)$ and so $\pi\left(P_{\mathbf{v}}[i]\right)$ and $\pi\left(P_{\mathbf{w}}[i]\right)$ are well-defined elements in $\mathbb{Z}\left[x_{1}^{ \pm 1}, x_{2}^{ \pm 1}\right]$.

We can thus give the following description of cluster variables in $\mathcal{A}(b, c)$ : 
Proposition 7. Let $b, c$ be positive integers. Then, for any $m \in \mathbb{Z}$, we have

$$
\begin{aligned}
& x_{2 m+1}=\pi\left(X_{P_{v}[m+1]}\right) \\
& x_{2 m+2}=\pi\left(X_{P_{w}[m+1]}\right)
\end{aligned}
$$

Proof. We prove it by induction on $m$. We have $x_{1}=\pi\left(u_{v_{i}}\right)=\pi\left(X_{P_{v_{i}}[1]}\right)$ for every $i=1, \ldots, b$ and $x_{2}=\pi\left(u_{w_{i}}\right)=\pi\left(X_{P_{w_{i}}[1]}\right)$ for every $i=1, \ldots, c$.

Fix $i \in\{1, \ldots, c\}$ and $m \in \mathbb{Z}$. It follows from $\left[\mathrm{BMR}^{+} 06\right]$ that indecomposable objects in the shift-orbit of a projective $k Q$-module have trivial endomorphism ring in $\mathcal{C}_{Q}$. Thus, we have isomorphisms of $k$-vector spaces

$$
\begin{aligned}
k & \simeq \operatorname{End}_{\mathcal{C}_{Q}}\left(P_{w_{i}}[m+1]\right) \\
& \simeq \operatorname{Hom}_{\mathcal{C}_{Q}}\left(P_{w_{i}}[m+1], P_{w_{i}}[m+1]\right) \\
& \simeq \operatorname{Hom}_{\mathcal{C}_{Q}}\left(P_{w_{i}}[m+1],\left(P_{w_{i}}[m]\right)[1]\right) \\
& \simeq \operatorname{Ext}_{\mathcal{C}_{Q}}^{1}\left(P_{w_{i}}[m+1], P_{w_{i}}[m]\right)
\end{aligned}
$$

where the last equality follows from the fact that $\mathcal{C}_{Q}$ is a triangulated category. Since, $\mathcal{C}_{Q}$ is 2-Calabi-Yau, we have an isomorphism of $k$-vector spaces

$$
k \simeq \operatorname{Ext}_{\mathcal{C}_{Q}}^{1}\left(P_{w_{i}}[m+1], P_{w_{i}}[m]\right) \simeq \operatorname{Ext}_{\mathcal{C}_{Q}}^{1}\left(P_{w_{i}}[m], P_{w_{i}}[m+1]\right) .
$$

The corresponding triangles are

$$
\begin{gathered}
P_{w_{i}}[m] \longrightarrow 0 \longrightarrow P_{w_{i}}[m+1] \longrightarrow P_{w_{i}}[m+1] \\
P_{w_{i}}[m+1] \longrightarrow \bigoplus_{j=1}^{c} P_{v_{j}}[m] \longrightarrow P_{w_{i}}[m] \longrightarrow P_{w_{i}}[m+2] .
\end{gathered}
$$

It thus follows from Theorem 4 that

$$
X_{P_{w_{i}}[m]} X_{P_{w_{i}}[m+1]}=\prod_{i=1}^{c} X_{P_{v_{j}}[m]}+1
$$

but $\pi\left(X_{P_{w_{i}}[m]}\right)=x_{2 m}$ and $\pi\left(X_{P_{v_{j}}[m]}\right)=x_{2 m-1}$ for every $j=1, \ldots, b$. We thus get

$$
\pi\left(X_{P_{w_{i}}[m+1]}\right)=\frac{x_{2 m-1}^{b}+1}{x_{2 m}}=x_{2 m+2} .
$$

The other cases are proved similarly.

Figure 1 sums up the situation where in the AR-quiver of $\mathcal{C}_{Q}$, we grouped together the objects in the same $G$-orbit and the arrows between these orbits. 


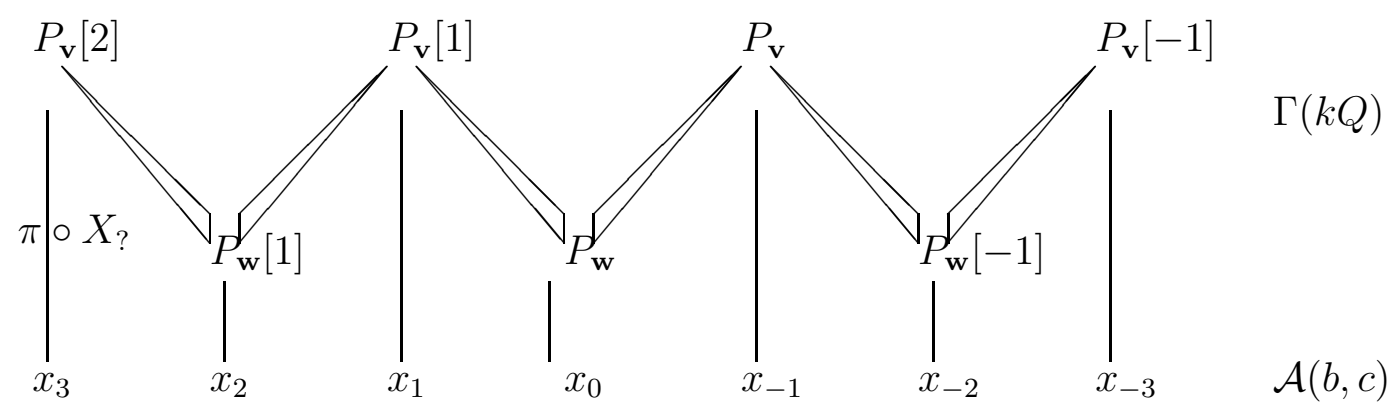

Figure 1: Realizing cluster variables

Locally, the quiver has actually the following shape:

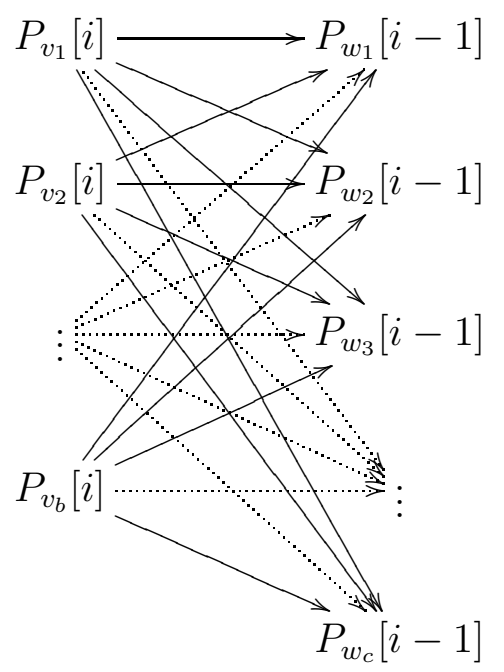

We now prove the main theorem:

Theorem 8. Let b, c be positive integers. With the above notations, we have

$$
\left\{x_{k}: k \in \mathbb{Z}\right\} \subset \mathbb{N}\left[x_{m}^{ \pm 1}, x_{m+1}^{ \pm 1}\right]
$$

for any $m \in \mathbb{Z}$.

Proof. Let $b, c$ be positive integers. All the exchange matrices in $\mathcal{A}(b, c)$ are either $\left[\begin{array}{rr}0 & b \\ -c & 0\end{array}\right]$ or $\left[\begin{array}{rr}0 & -b \\ c & 0\end{array}\right]$. It is thus enough to prove that cluster variables in $\mathcal{A}(b, c)$ can be expressed as substraction-free expressions in the initial cluster $\mathbf{c}=\left(x_{1}, x_{2}\right)$.

Fix a cluster variable $x$ in $\mathcal{A}(b, c)$. Then $x=x_{k}$ for some $k \in \mathbb{Z}$. Assume for example that $k=2 m+2$ for some $m \in \mathbb{Z}$. Then it follows from Proposition 7 that $x=$ 
$\pi\left(X_{P_{\mathbf{w}}[m+1]}\right)$. According to Theorem 5, as $K_{b, c}$ is acyclic, $X_{P_{\mathbf{w}}[m+1]}$ is a linear combination of Laurent monomials in $\mathbf{u}$ with positive coefficients. Thus, applying $\pi$ to this expansion, $x=\pi\left(X_{P_{\mathbf{w}}[m+1]}\right)$ is a linear combination of Laurent monomials in $\left(x_{1}, x_{2}\right)$ with positive coefficients. Similarly, if $k=2 m+1, x_{k}$ can be written as a linear combination of Laurent monomials in $\left(x_{1}, x_{2}\right)$ with positive coefficients. This proves the theorem.

Example 9. We consider the cluster algebra $\mathcal{A}(2,3)$ with initial cluster $\left(x_{1}, x_{2}\right)$. We consider the following quiver:

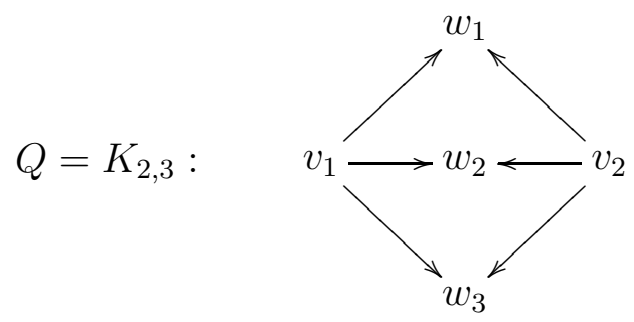

Let $\mathcal{A}(Q)$ be the cluster algebra with initial seed $\left(B_{Q}, \mathbf{u}\right)$ where

$$
\mathbf{u}=\left\{u_{v_{1}}, u_{v_{2}}, u_{w_{1}}, u_{w_{2}}, u_{w_{3}}\right\} .
$$

Let $X_{\text {? }}: \mathrm{Ob}\left(\mathcal{C}_{Q}\right) \longrightarrow \mathbb{Z}\left[\mathbf{u}^{ \pm 1}\right]$ be the corresponding Caldero-Chapoton map.

A direct computation gives

$$
\begin{gathered}
X_{P_{w_{j}}}=\frac{1+u_{v_{1}} u_{v_{2}}}{u_{w_{j}}} \\
X_{I_{w_{j}}}=X_{P_{w_{j}}[2]}=\frac{1+u_{v_{1}} u_{v_{2}}+2 u_{w_{1}} u_{w_{2}} u_{w_{3}}+u_{w_{1}}^{2} u_{w_{2}}^{2} u_{w_{3}}^{2}}{u_{w_{j}} u_{v_{1}} u_{v_{2}}}
\end{gathered}
$$

for every $j=1,2,3$ and

for every $i=1,2$.

$$
\begin{gathered}
X_{P_{v_{i}}}=\frac{1+u_{v_{1}}^{3} u_{v_{2}}^{3}+3 u_{v_{1}}^{2} u_{v_{2}}^{2}+3 u_{v_{1}} u_{v_{2}}+u_{w_{1}} u_{w_{2}} u_{w_{3}}}{u_{v_{i}} u_{w_{1}} u_{w_{2}} u_{w_{3}}}, \\
X_{I_{v_{i}}}=X_{P_{v_{i}}[2]}=\frac{1+u_{w_{1}} u_{w_{2}} u_{w_{3}}}{u_{v_{i}}}
\end{gathered}
$$

Consider the folding morphism

$$
\pi:\left\{\begin{array}{rlrl}
\mathbb{Z}\left[u_{i}^{ \pm 1}: i \in Q_{0}\right] & \longrightarrow \mathbb{Z}\left[x_{1}^{ \pm 1}, x_{2}^{ \pm 1}\right] & \\
u_{v_{i}} & \mapsto x_{1} & & \text { for all } i=1,2 ; \\
u_{w_{j}} & \mapsto x_{2} & & \text { for all } j=1,2,3 .
\end{array}\right.
$$

Thus we get

$$
\begin{gathered}
\pi\left(X_{P_{v_{i}}}\right)=\frac{1+x_{1}^{6}+3 x_{1}^{4}+3 x_{1}^{2}+x_{2}^{3}}{x_{1} x_{2}^{3}}=x_{-1}, \quad \pi\left(X_{P_{w_{j}}}\right)=\frac{1+x_{1}^{2}}{x_{2}}=x_{0}, \\
\pi\left(X_{P_{v_{i}}[1]}\right)=x_{1}, \quad \pi\left(X_{P_{w_{j}}[1]}\right)=x_{2}, \\
\pi\left(X_{I_{v_{i}}}\right)=\frac{1+x_{2}^{3}}{x_{1}}=x_{3}, \quad \pi\left(X_{I_{w_{j}}}\right)=\frac{1+x_{1}^{2}+2 x_{2}^{3}+x_{2}^{6}}{x_{1}^{2} x_{2}}=x_{4} .
\end{gathered}
$$

This illustrates Theorem 8. 


\section{References}

[BFZ05] A. Berenstein, S. Fomin, and A. Zelevinsky. Cluster algebras III: Upper bounds and double Bruhat cells. Duke Mathematical Journal, 126(1):1-52, 2005. MR2110627 (2005i:16065).

$\left[\mathrm{BMR}^{+} 06\right]$ A. Buan, R. Marsh, M. Reineke, I. Reiten, and G. Todorov. Tilting theory and cluster combinatorics. Advances in mathematics, 204(2):572-618, 2006. MR2249625 (2007f:16033).

[CC06] P. Caldero and F. Chapoton. Cluster algebras as Hall algebras of quiver representations. Commentarii Mathematici Helvetici, 81:596-616, 2006. MR2250855 (2008b:16015).

[CK06] P. Caldero and B. Keller. From triangulated categories to cluster algebras II. Annales Scientifiques de l'Ecole Normale Supérieure, 39(4):83-100, 2006. MR2316979 (2008m:16031).

[CK08] P. Caldero and B. Keller. From triangulated categories to cluster algebras. Inventiones Mathematicae, 172:169-211, 2008. MR2385670.

[CR08] P. Caldero and M. Reineke. On the quiver grassmannian in the acyclic case. Journal of Pure and Applied Algebra, 212(11):2369-2380, 2008. MR2440252.

[Dem08a] L. Demonet. Catégorifications d'algèbres amassées antisymétrisables. $\mathrm{PhD}$ thesis, Université de Caen, november 2008.

[Dem08b] L. Demonet. Cluster algebras and preprojective algebras : the non simplylaced case. C. R. Math. Acad. Sci. Paris, 346(7-8):379-384, 2008. MR2417554.

[Dup08] G. Dupont. An approach to non simply laced cluster algebras. Journal of Algebra, 320(4):1626-1661, 2008. MR2431998.

[FZ02] S. Fomin and A. Zelevinksy. Cluster algebras I: Foundations. J. Amer. Math. Soc., 15:497-529, 2002. MR1887642 (2003f:16050).

[FZ03] S. Fomin and A. Zelevinksy. Cluster algebras II: Finite type classification. Inventiones Mathematicae, 154:63-121, 2003. MR2004457 (2004m:17011).

[FZ07] S. Fomin and A. Zelevinksy. Cluster algebras IV: Coefficients. Composition Mathematica, 143(1):112-164, 2007. MR2295199 (2008d:16049).

[Kel05] B. Keller. On triangulated orbit categories. Documenta Mathematica, 10:551581, 2005. MR2184464 (2007c:18006).

[MP06] G. Musiker and J. Propp. Combinatorial interpretations for rank-two cluster algebras of affine type. Electron. J. Combin., 14, 2006. MR2285819 (2008j:05374).

[SZ04] P. Sherman and A. Zelevinsky. Positivity and canonical bases in rank 2 cluster algebras of finite and affine types. Mosc. Math. J., 4:947-974, 2004. MR2124174 (2006c:16052). 